\title{
Low-Actuation-Voltage MEMS for 2-D Optical Switches
}

\author{
Hsin-Ta Hsieh, Chen-Wei Chiu, Tom Tsao, Fukang Jiang, and Guo-Dung John Su, Member, IEEE
}

\begin{abstract}
This paper discusses the design, fabrication, and test results of electromagnetically actuated two-dimensional (2-D) microelectromechanical systems (MEMS) optical switches. The switching element consists of a $20 \mu \mathrm{m} \times 500 \mu \mathrm{m} \times 1200 \mu \mathrm{m}$ vertical micromirror, which is monolithically integrated with an actuation flap. The micromirror is made by anisotropic tetramethyl-ammonium-hydroxide wet etching with an optical insertion loss of about $0.2 \mathrm{~dB}$. A maximum insertion loss of $2.1 \mathrm{~dB}$ has been experimentally demonstrated for a $10 \times 102-\mathrm{D}$ optical crossconnect switch. The actuation flap has double layers of spiral metal coils to generate a large actuation force with the permanent magnets placed at the bottom of the MEMS chip. The magnetic flux is created on the surface of a pair of opposite polarized magnets to precisely control the moving direction of the vertical mirror. The required voltage is less than $0.5 \mathrm{~V}$, and the power consumption is about $3.5 \mathrm{~mW}$ for a switching element. Due to the center symmetric design and the stress-free characteristic of the micromirror, the temperature dependence loss is demonstrated to be as low as $0.05 \mathrm{~dB}$. A switching time of $5 \mathrm{~ms}$ is achieved by applying the proper driving waveform.
\end{abstract}

Index Terms-Electromagnetic force, free space, low voltage, optical crossconnect (OXC) switches, optical microelectromechanical systems (MEMS), vertical micromirrors.

\section{INTRODUCTION}

D URING the past few years, broadband communications and wireless access have made explosive progress in Internet data traffic, which required the evolution of opticaltelecommunications networks for simple, quick, and flexible applications. As the data bit rate increases, it will be more difficult and expensive to implement an electronic switching fabric solution, which is also known as O-E-O. Optical crossconnect (OXC) switching technology provides a promising solution to realize high-capacity and high-complexity systems in the future. Transparent space-division optical switches are needed for rerouting optical signals in fiber networks. The requirements of such switches include low insertion loss, low crosstalk, and wavelength independence. Microelectromechanical systems (MEMS) have emerged as a leading candidate for achieving true all-optical wavelength routing [1], [2]. Leveraging the inherent advantages of MEMS such as batch fabrication process, small size, and scalability, MEMS optical switches with proper

Manuscript received January 17, 2006; revised July 7, 2006. This work was supported in part by the National Science Council of Taiwan under Grant NSC 94-2112-M-002-042.

H.-T. Hsieh and G.-D. J. Su are with the Graduate Institute of Electro-Optical Engineering and the Department of Electrical Engineering, National Taiwan University, Taipei 106, Taiwan, R.O.C. (e-mail: r93941031@ntu.edu.tw; gdjsu@cc.ee.ntu.edu.tw).

C.-W. Chiu, T. Tsao, and F. Jiang are with Umachines, Inc., Altadena, CA 91001 USA.

Digital Object Identifier 10.1109/JLT.2006.883674 designs could provide reliability, repeatability, and reasonably fast-switching speed for optical-communication applications.

One can distinguish between two MEMS approaches for optical switching: two-dimensional (2-D, digital) and threedimensional (3-D, analog) MEMS. Two-dimensional MEMS technology shows promising progress for all-optical network applications since it allows lower cost per switching port [3]. Unlike the 3-D MEMS approach, which tilts micromirrors analogically to establish connection paths to make large-port-count switches with more than 1000 input and output ports, the mirror position in the 2-D configuration is bistable. Therefore, the driving mechanism for the 2-D MEMS switches is digital and straightforward, which makes the 2-D MEMS suitable for lowport-count applications [4]-[8]. In addition to large switches, small switches with port counts ranging from 2 to 32 ports are also used by optical networks. These small-scale switches are used in optical add/drop multiplexers as well as in-line systems for network-restoration applications [4]. Furthermore, by arranging 2-D OXC switches in a Clos network, largesize crossconnects can be built [5]. Digital MEMS switches, however, involve more design considerations on the MEMS devices. First, the angular tolerance for digital MEMS switches is very tight. An angular misalignment of $0.1^{\circ}$ might cause up to $2-\mathrm{dB}$ additional loss for the $200-\mu \mathrm{m}$ beam waist [6]. Vertical micromirrors were proposed to solve this difficulty so that the surface normal of micromirrors remains constant as the micromirrors are inserted into the optical paths [7], [8]. However, it is difficult to fabricate high-quality vertical micromirrors due to high-aspect-ration challenges. Deep reactive ion etching (DRIE) is applied to make vertical structures, but the etching depth is limited by mask openings, and the sidewalls may suffer ripples that cause extra optical loss. Vertical mirrors can be made alternatively by rotating thin films of polysilicon with appropriate designs of microhinges by $90^{\circ}$. Unfortunately, micromirrors made with deposited thin films need extra care to balance residual stress inside the films so that buckling of micromirrors can be reduced [9]. In addition to the angular dependence, longitudinal misalignment is usually observed in digital MEMS optical switches since the optical beam diverges from the finite-emitting aperture of fiber collimators. Generally speaking, the larger the beam diameter, the smaller the insertion-loss variation for different optical path lengths. As a result, large micromirrors are preferred to accommodate large beam diameters on the order of a few hundreds of micrometers. This requires microactuators to travel long distances and favors the electromagnetic actuation force from the point of view of energy density [10]. In other words, the electromagnetic actuation method needs much less actuation voltage to move 


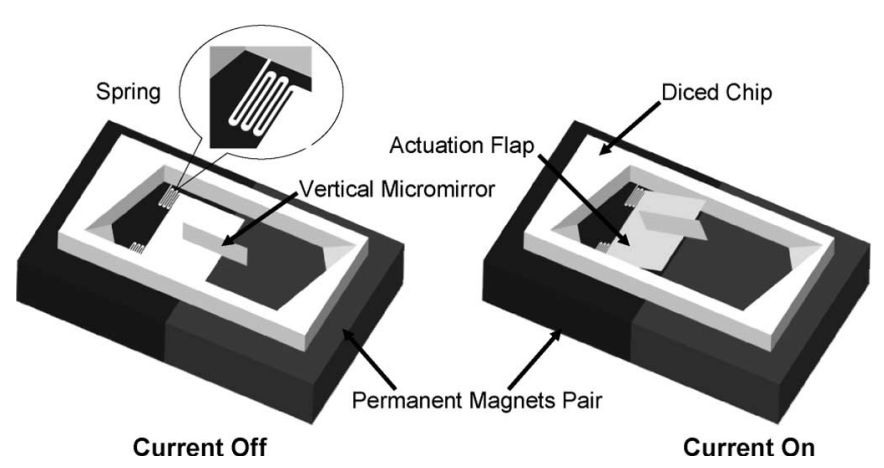

Fig. 1. Schematic drawing of a vertical micromirror for 2-D optical switches.

micromirrors than the popular electrostatic method, which typically ranges from a few tens to hundreds of volts. Therefore, the magnetic circuit design is important for the electromagnetic actuation method to ensure uniform magnetic flux over all of the switching matrixes [11].

This paper started with the design and fabrication of an electromagnetically actuated MEMS switch, which consists of a vertical micromirror and an actuation flap with patterned metal coils. By carefully considering the corner compensation, vertical micromirrors were fabricated on the (110) silicon wafer. The vertical micromirror is shaped and smoothed by anisotropic tetramethyl ammonium hydroxide (TMAH) wet etching in one step to reduce fabrication cost and make high-quality micromirrors. The surface area of micromirrors made by TMAH is $500 \mu \mathrm{m} \times 1200 \mu \mathrm{m}$, and the surface roughness could be as low as $20 \mathrm{~nm}$. The interaction between the electric currents through the metal coils of the actuation flap and the permanent magnets placed underneath the MEMS chips results in a large actuation force, which requires only $0.5 \mathrm{~V}$ to move the micromirrors completely into the optical paths. The optical insertion loss caused by the micromirrors is about $0.2 \mathrm{~dB}$. Chips consisting of $10 \times 10$ arrays are successfully fabricated. The magnetic circuit designs presented in this paper could be scaled to larger arrays, and the proposed 2-D MEMS optical switches are experimentally demonstrated. Finally, technical comments conclude the paper in the final section. Compared with a previously published work [8], the proposed design could be operated at lower voltage $(0.5 \mathrm{~V})$. The large vertical micromirrors are fabricated by anisotropic TMAH wet etching to ensure low insertion loss $(\sim 0.2 \mathrm{~dB})$ at a relatively low cost [7], [11]. In addition, the temperature dependence loss (TDL) is measured to be as low as $0.05 \mathrm{~dB}$ due to its center symmetric configuration.

\section{DESIGn AND FABRICATION}

A schematic drawing of a switching element is plotted in Fig. 1. A vertical single-crystalline silicon (SCS) mirror is attached to the actuation flap to switch the light into the selected channel. The micromirror is stress free to ensure surface flatness and is independent of temperature changes. The switch is actuated by the electromagnetic force generated by the metal coils on the actuation flap and the external permanent magnets. The metal coils are patterned on the other side of the micromirror, and the diced chip is placed on the top of

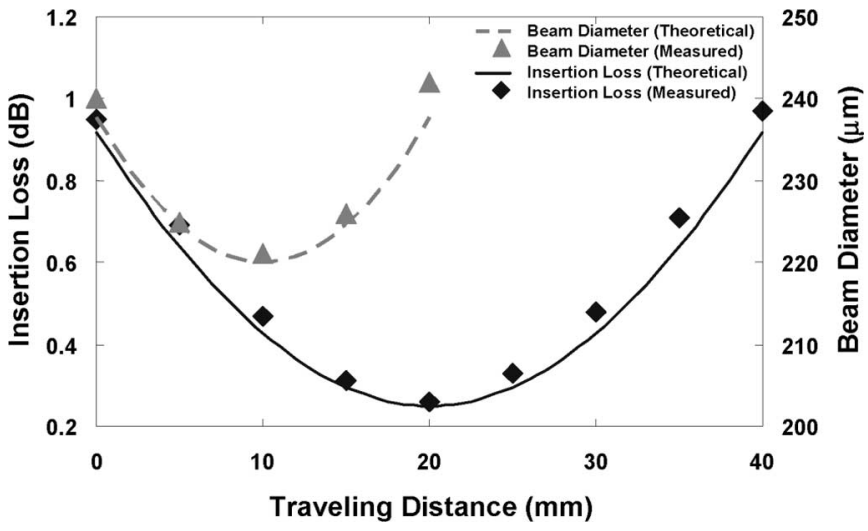

Fig. 2. Longitudinal misalignment loss and beam diameter versus traveling distance.

the magnets, as shown in Fig. 1. When the current is applied to the metal coils on the actuation flap, the electromagnetic force moves the SCS mirror out of the etched silicon cavity to reflect light. The moving direction of the silicon micromirror is perpendicular to the collimated lights to minimize angular misalignment. The micromirror is $20 \mu \mathrm{m}$ thick, $500 \mu \mathrm{m}$ high, and $1200 \mu \mathrm{m}$ long to ensure low optical insertion. The chip size of an $8 \times 8$ optical switch is $20 \mathrm{~mm} \times 20 \mathrm{~mm}$. Longitudinal misalignment accounts for a major optical power loss in 2-D optical switching due to the beam divergence from a finite-emitting aperture of fiber collimators. Our theoretical and experimental results in Fig. 2 show that the optical loss from a $220-\mu \mathrm{m}$ beam waist over the traveling difference of $\pm 20 \mathrm{~mm}$ from its optimized spot is $1 \mathrm{~dB}$, which ensures a safe margin for an $8 \times 8$ optical switch, as suggested by Telcordia GR1073 [12]. The longest distance before the collimated light hits a micromirror is $20 \mathrm{~mm}$. The truncation ratio of the mirror size and beam diameter is slightly larger than two to ensure good beam-energy coverage. In addition, vertical micromirrors could save the precious wafer surface area, and the mirror size can be increased without adding the light traveling distance, such as surface micromachining switches. In order to eliminate any possible stiction, the design does not use any mechanical stopper in the moving path of the micromirror. The microactuation flap is attached to the silicon sidewall with serpentine springs, which have a wider and rounded corner design to relieve stress concentration during the mechanical-shock up to $500 \mathrm{G}$ [13].

Metal coils on the actuation flap were employed to move the vertical micromirror electromagnetically. The energy density of the electromagnetic force is much larger than the popular electrostatic force so that no excessive high voltage is needed in the electromagnetic actuation [10]. In other words, electromagnetic force is more suitable than electrostatic force for moving the micromirror out of the light-path plane, which is in the range of a few hundreds of micrometers. The main difficulty of using electromagnetic actuation in MEMS is the magnetic circuit design. In our case, we proposed to use "a pair of opposite polarized magnets" design to provide a large and uniform magnetic force over the metal-coil-patterned actuation flap, as shown in Fig. 3. The north pole (denoted as magnet $+Z$ ) and south pole (denoted as magnet $-Z$ ) of the permanent magnets are aligned with each other on the same plane, which is 


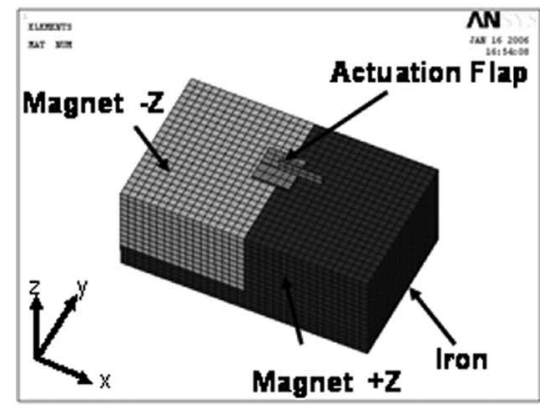

(a)

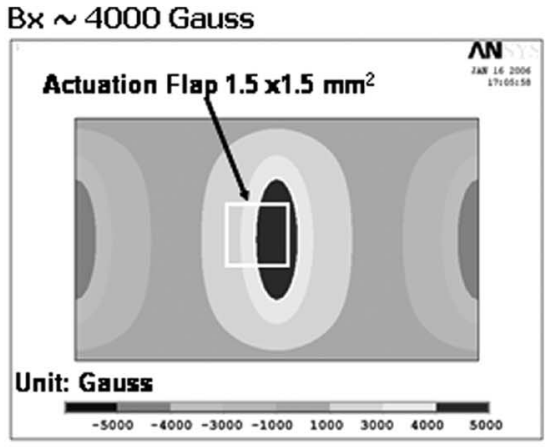

(b)

By 20 Gauss

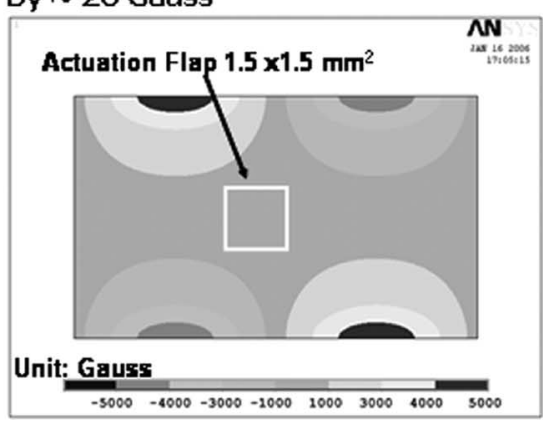

(c)

Fig. 3. Calculated magnetic field for a MEMS 2-D switch. (a) Model of the magnetic circuit. (b) Magnetic flux strength along the vertical mirror. (c) Magnetic flux strength perpendicular to the vertical mirror.

underneath the MEMS actuation flap. The size of each magnet is $3 \mathrm{~mm}$ in the $z$-direction, $6 \mathrm{~mm}$ in the $y$-direction, and $5 \mathrm{~mm}$ in the $x$-direction so that the total size of the magnet pair is $3 \times 6 \times 10 \mathrm{~mm}^{3}$. An iron plate with 1-mm thickness is placed underneath the magnets to reduce magnetic flux leakage to the perimeters. The magnetic flux comes from the north pole and enters the south pole. This provides the necessary magnetic flux in line with the vertical micromirror, which is defined as the $x$-direction. The magnetic flux along the $x$-direction $B_{x}$ causes the pitching movement of the actuation flap. On the other hand, the magnetic flux along the $y$-direction $B_{y}$ could introduce undesired rolling and yawing of the actuation flap. Fig. 3 shows the simulated magnetic flux, which is $750 \mu \mathrm{m}$ above the magnetic surface, around the actuation flap. A uniform field of $4000 \mathrm{G}$ is presented to actuate the MEMS switch in the pitching direction; meanwhile, the magnetic field for the yaw and roll direction is minimized to reduce potential angular misalignment. The magnitude of $B_{x}$ is about 200 times larger than that of $B_{y}$. In addition, the serpentine springs connected to the actuation flap are more compliant in the pitching mode to further suppress the angular misalignment for fiber coupling loss. This design can be expanded into a larger matrix design, as shown in Fig. 4 , in which a $4 \times 4$ switch chip is placed on an eight-magnet ribbon design. The magnetic flux can be maintained without losing its strength as the matrix expands.

To fabricate MEMS optical switches with smooth and vertical reflecting surfaces, we started with 4-in double-sided polished silicon-on-insulator wafers with a (110) substrate and a (100) device layer of $10 \mu \mathrm{m}$, which were fusion bonded together. A low-temperature-oxidation silicon dioxide of $0.7 \mu \mathrm{m}$

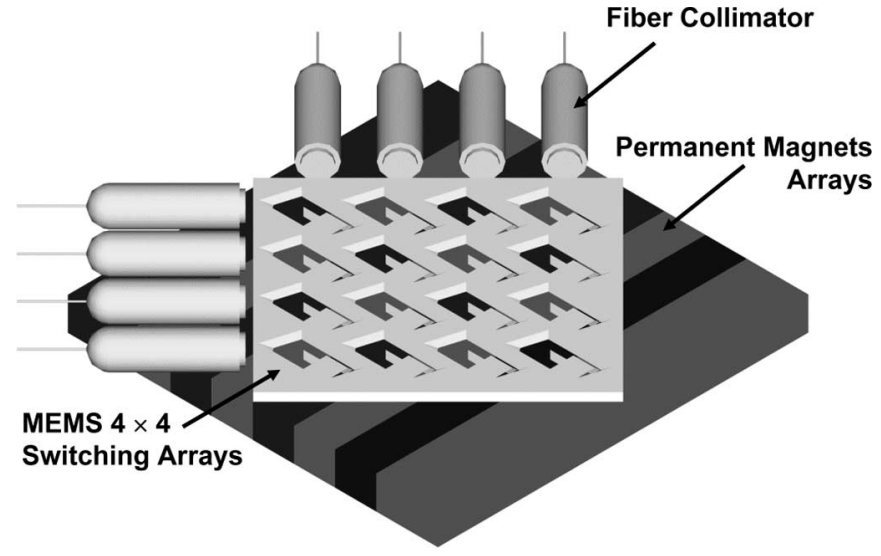

Fig. 4. Schematic drawing of a $4 \times 4$ MEMS optical switch with periodical magnet ribbons.

was first deposited and patterned as wet-etching masks. Using anisotropic TMAH wet etching, vertical walls were created on the (111) planes of the (110) substrate wafer and were used as the reflecting mirror, as shown in step 2 of Fig. 5. In general, TMAH is more IC compatible, less toxic, and more stable [14] than other alkaline-based wet etchant, such as potassium hydroxide. SCS micromirrors made by wet etching are stress free and flat due to crystal orientation. The cost of wet etching is relatively low compared with other MEMS etching processes for making high-aspect-ration structures, such as DRIE, and the etching results are predictable and repeatable. After the vertical mirrors were made, two layers of chromium $(100 \AA) /$ gold $(1 \mu \mathrm{m})$ were deposited by an e-beam evaporator on the device layers and were patterned to make metal coils to generate 

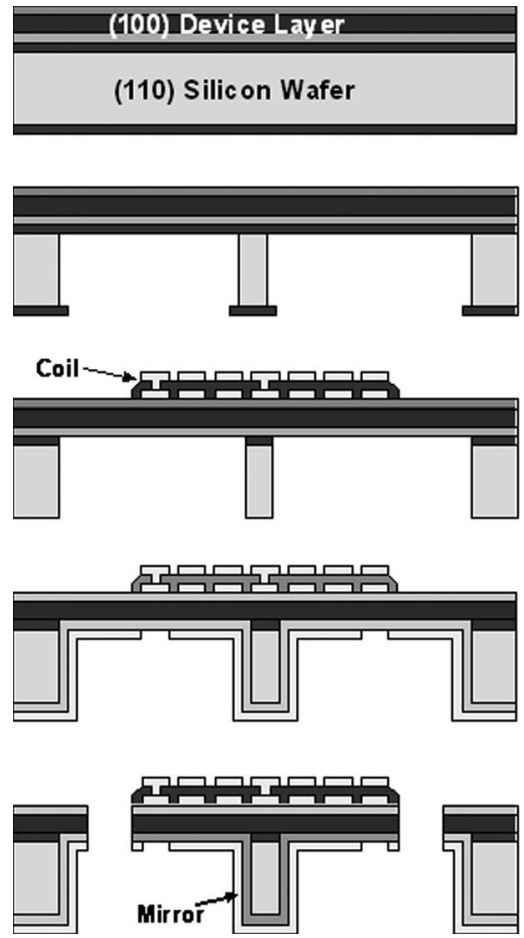

Fig. 5. Fabrication process of 2-D optical MEMS switches.

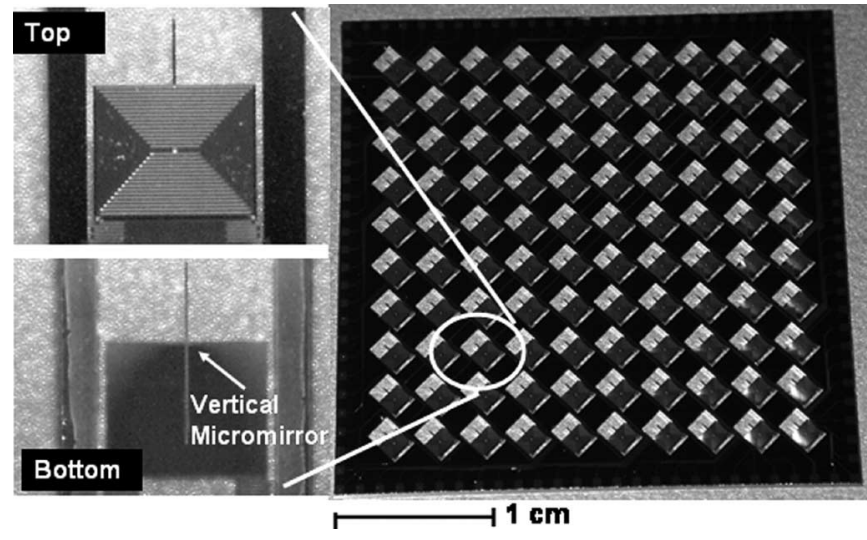

Fig. 6. Fabricated $10 \times 10$ switch array.

electromagnetic force, as shown in step 3. There is an insulation layer of $1-\mu \mathrm{m}$ plasma enhanced chemical vapor deposition (PECVD) low-stress nitride between the two metal coils. Then, the flaps and serpentine springs were patterned by DRIE on the device layer to release microactors in the final step. The complete fabrication process flow is illustrated in Fig. 5. The picture of a $10 \times 10$ switch array is shown in Fig. 6 with zoomed pictures of a single switching element. The (111) surface plane can be clearly seen as the vertical micromirror. A corner compensation design is necessary in the mask drawing, and the mirror length is controlled by time etching. The fabricated micromirror is $20 \mu \mathrm{m}$ wide, $1200 \mu \mathrm{m}$ long, and $500 \mu \mathrm{m}$ high.

\section{EXPERIMENTAL TEST RESUlts AND DisCUSSION}

The surface roughness of the SCS micromirror is measured by optical interferometer from WYKO. The root-mean-square
(1). Start with (110) wafer fusion bonded with a (100) device layer

(2). Wet etching on the bottom side of the (110) wafer to create vertical micromirror

(3). Pattem the metal coils on the top side of the (100) device layer

(4). Coat the gold on the vertical mirror

(5). Pattern the actuation flap and torsion springs on the (100) device layer to release switches

surface roughness of micromirrors could be as low as $20 \mathrm{~nm}$. The scattering light caused by the micromirror due to surface roughness is estimated [6] as follows:

$$
P_{\text {scat }}=\left(1-e^{-\left(4 \pi \sigma \cos \theta_{i} / \lambda\right)^{2}}\right) P_{\text {tot }}
$$

where $P_{\text {tot }}$ is the total flux of the incident light, $P_{\text {scat }}$ is the flux of the light scattered, $\lambda$ is the wavelength, $\theta_{i}$ is the incident angle, and $\sigma$ is the surface roughness. The optical insertion loss was measured using a pair of commercial (from NSG) single-mode fiber collimators with a GRIN lens attached to a fiber. The GRIN lens is antireflection coated at $1.55 \mu \mathrm{m}$, and the fiber collimators are mounted on the five-axis precision alignment stage. The beam diameter $\left(1 / e^{2}\right)$ from the fiber collimator is about $240 \mu \mathrm{m}$, which can almost be covered by the vertical micromirror $(500 \mu \mathrm{m} \times 1200 \mu \mathrm{m})$. The insertion losses of the micromirrors were measured using 40 pieces to study the statistic distribution of the surface quality. It was found that the insertion loss fell into the range of $0.2 \pm 0.15 \mathrm{~dB}$, which is close to the theoretical calculation in (1). Fig. 7 shows the insertion loss and the corresponding surface roughness versus the numbers of chips. The chips are randomly picked from different wafers at various locations, and it is observed that there was no strong correlation between chip location and surface roughness. We assumed that surface roughness is more related to impurities and crystal imperfection due to silicon ingot growth.

To achieve high optical coupling efficiencies, the alignment of external optical components to the chip has to be as precise as possible. The surface normal of vertical micromirrors is uniform because it is aligned to the silicon crystal orientation. This reduces the effects of angular misalignment. In addition, insertion loss caused by surface flatness has been experimentally proved to be low. We assume that longitudinal misalignment 


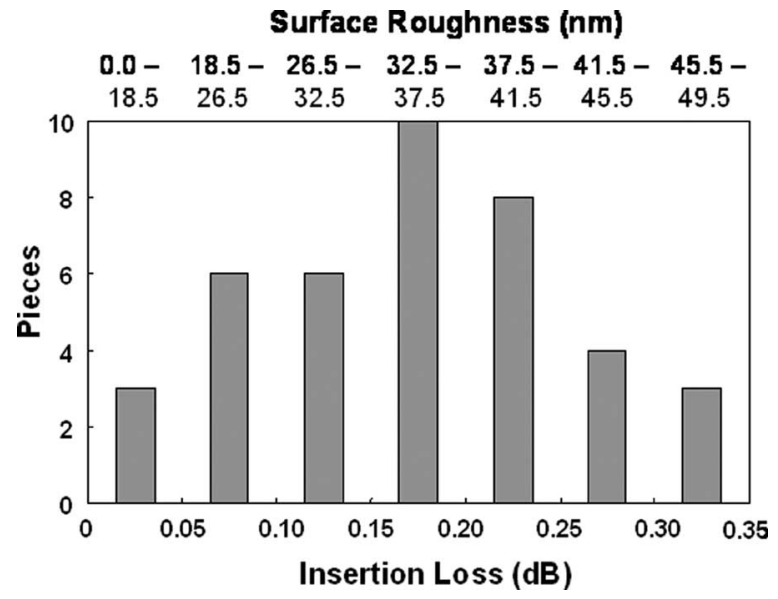

Fig. 7. Insertion loss and surface roughness of vertical micromirrors.

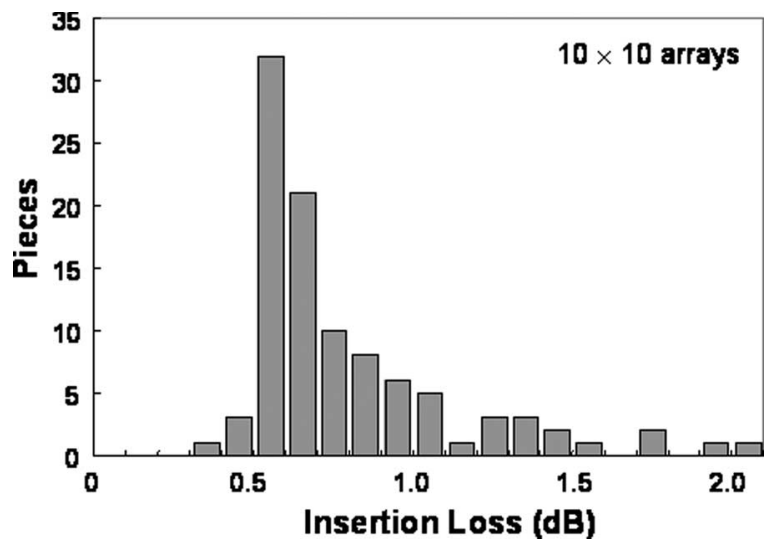

Fig. 8. Insertion-loss distribution of a fabricated $10 \times 10$ switch.

will be a determining factor to optical insertion loss, and the coupling efficiency is a function of coupling length. The collimated optical beams diverge as they propagate through free space due to the finite-emitting beam aperture. This imposes fundamental limits on optical coupling efficiency. By applying Gaussian-shaped mode, we noticed that coupling loss drops rapidly as the mirror and beam sizes increase, suggesting that beam divergence is the dominant factor in this region. The effect saturates for mirror radii that are larger than $500 \mu \mathrm{m}$. This is based on a truncation ratio of 0.5 , which means that the waist diameters of a Gaussian beam from a fiber collimator is half the size of the micromirror. Fiber-micromirror-fiber losses of $2.1 \mathrm{~dB}$ were measured through the longest path of the $10 \times 10$ switch array. Fig. 8 shows the insertion-loss distribution of a fabricated $10 \times 10$ optical switch. The crosstalk is almost negligible $(<-50 \mathrm{~dB})$ in our measurement over the entire $10 \times 10$ array. Considering our fabrication processes on a 4-in silicon wafer and the optical beam divergence, we assume that a $16 \times 16$ optical switch will be a practical limit. The estimated maximum insertion loss for a $16 \times 16$ array is about $3 \mathrm{~dB}$, based on the result from the $10 \times 10$ switch previously discussed.

The switching is achieved by passing the current through the metal coils on the actuation flap to move the micromirror up and down, as shown in Fig. 9(a). The required voltage to move the entire micromirror out of the silicon cavity is $0.5 \mathrm{~V}$, typically with a current of $7 \mathrm{~mA}$. Fig. 9(b) shows the normalized

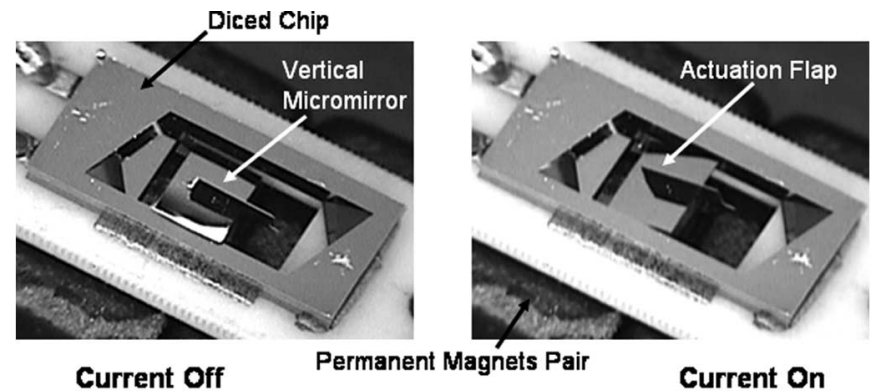

(a)

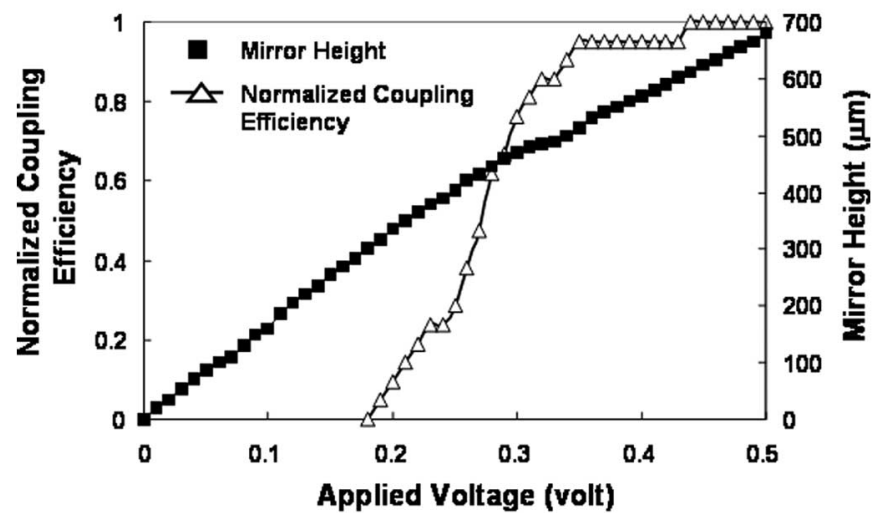

(b)

Fig. 9. (a) Operated device and (b) applied voltage versus normalized coupling efficiency and moving heights.

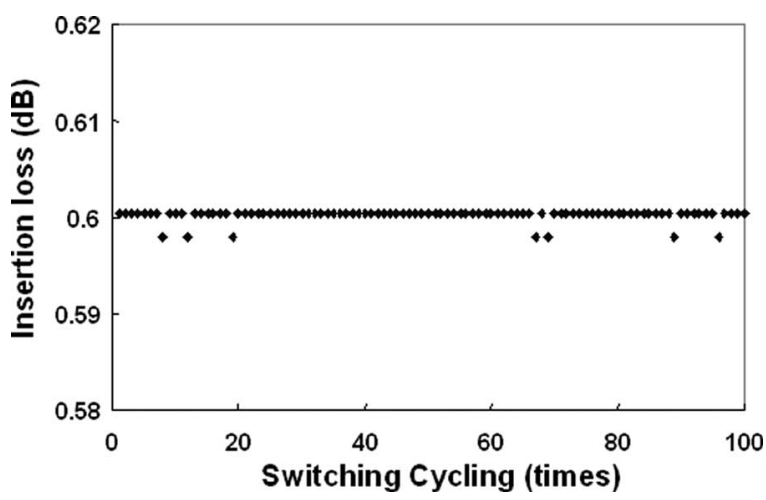

Fig. 10. Repeatability of a 2-D MEMS switch.

fiber-micromirror-fiber coupling efficiency and mirror heights against the applied voltage. The mirror height is almost linear to the applied voltage. Because of the vertical-mirror configuration, the highest coupling efficiency is achieved within a mirror-height tolerance of $100 \mu \mathrm{m}$. This is a very important characteristic to realize good switch repeatability because the optimized fiber coupling efficiency is less dependent on the final position of the reflecting surface, as plotted in Fig. 10. The measurement is performed over 100 consecutive switching cycles. Due to the lack of a mechanical stopper, repeatability is a critical issue to this design for purposes of digital MEMS switching. Fortunately, a large mirror surface can tolerate vertical movement in a relatively large range of $100 \mu \mathrm{m}$, and repeatability is within the range of $0.01 \mathrm{~dB}$.

The TDL is a challenge for MEMS optical switches because the insertion loss is very sensitive to angular misalignment. The 


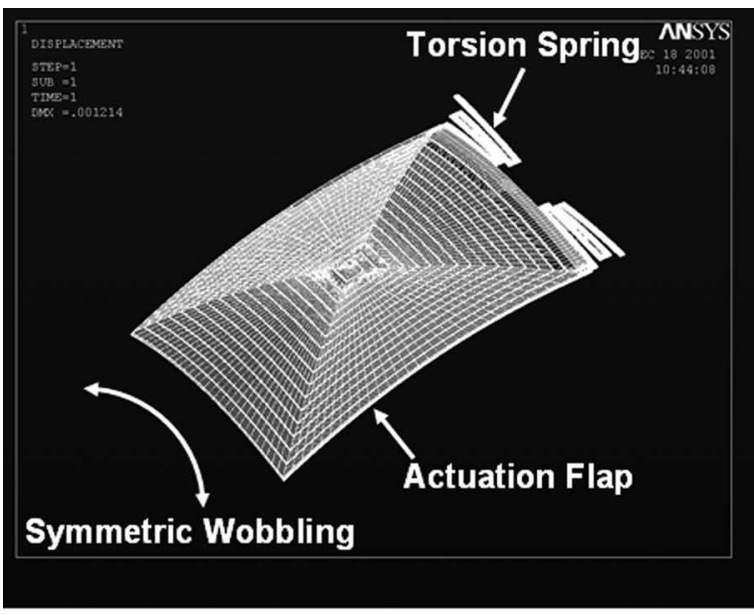

(a)

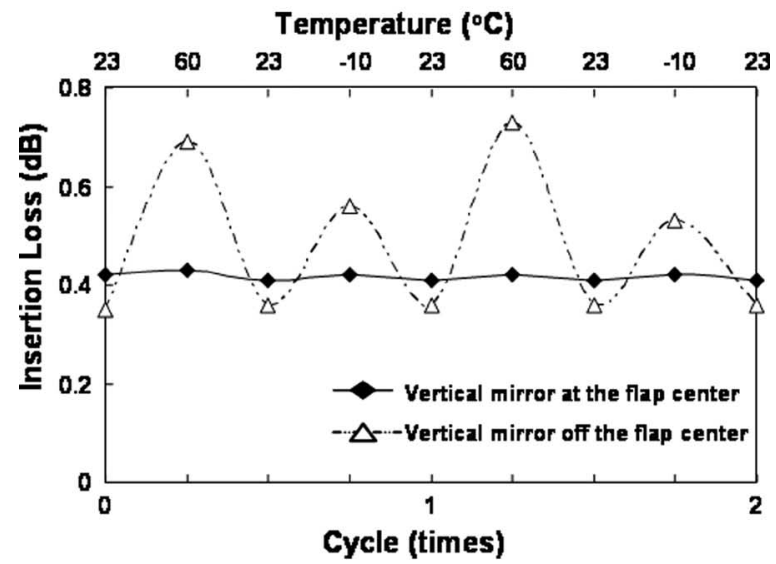

(b)

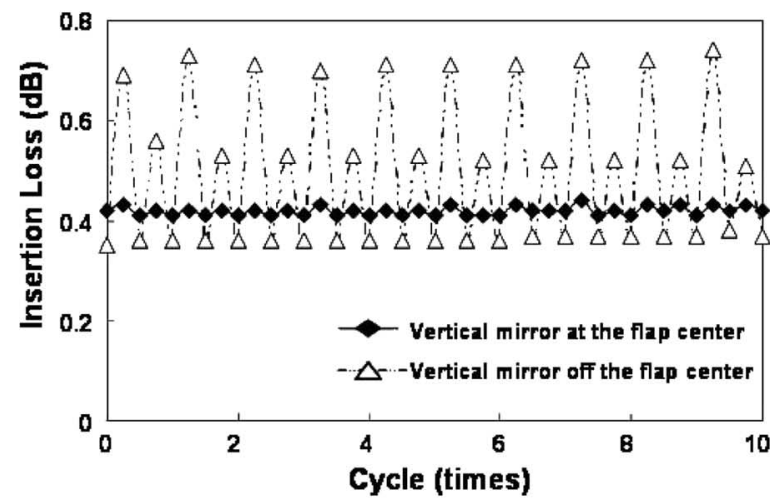

(c)

Fig. 11. (a) Finite-element modeling of the actuation flap at an escalated temperature. (b) TDL of the first two cycles. (c) TDL of ten cycles for a micromirror.

vertical mirror is made by stress-free SCS and is very stable in the range of $-10{ }^{\circ} \mathrm{C}$ to $60{ }^{\circ} \mathrm{C}$. In our design, we found that the TDL is susceptible to the micromirror position in the actuation flap, as illustrated in Fig. 11(a). Indeed, the actuation flap is a perfect bimorph structure, and the deformation follows the coefficient-of-thermal-expansion mismatch between the gold layer and silicon thin film. To investigate TDL, we measured four data points at different temperatures $\left(23^{\circ} \mathrm{C}, 60^{\circ} \mathrm{C}, 23^{\circ} \mathrm{C}\right.$, and $-10{ }^{\circ} \mathrm{C}$ ) in each cycle. The measurement of the first two temperature cycles is shown in Fig. 11(b) to illustrate the

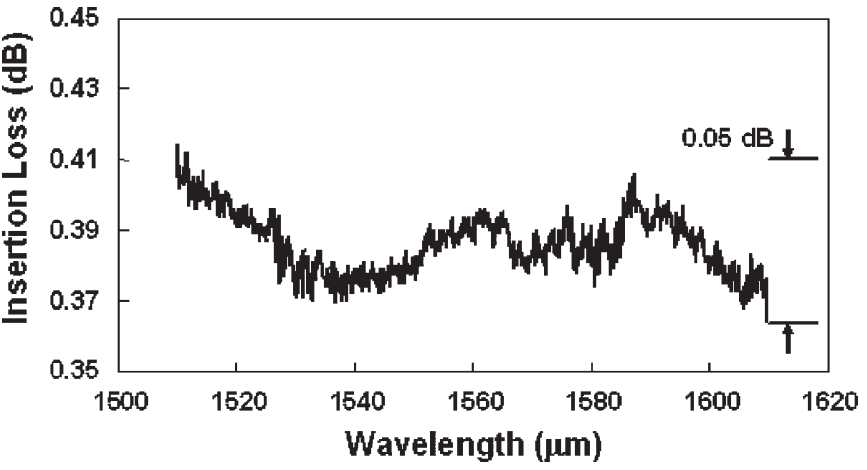

Fig. 12. WDL of a vertical micromirror.

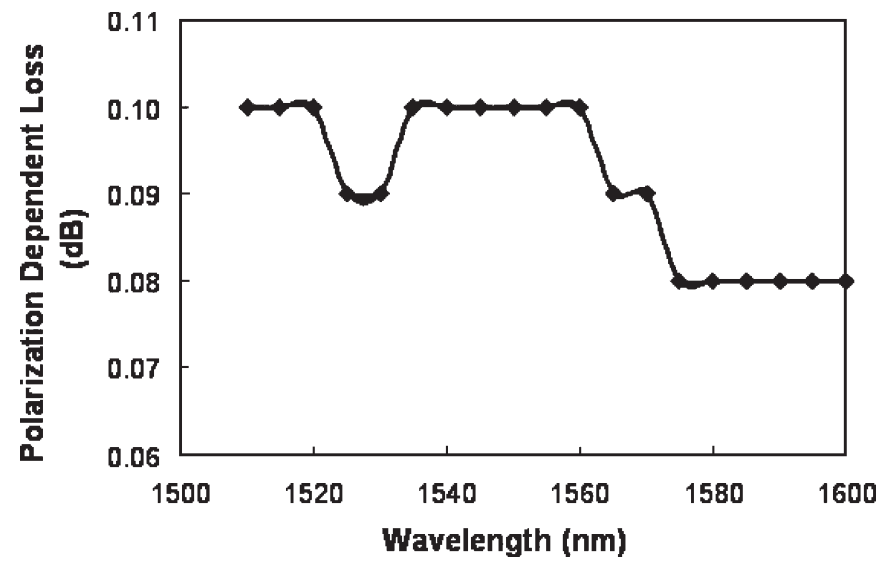

Fig. 13. PDL of a vertical micromirror.

insertion loss at different temperatures. The optical alignment is performed at room temperature so that the insertion loss is lowest at $23{ }^{\circ} \mathrm{C}$ and is about $0.4 \mathrm{~dB}$. The insertion loss is increased to 0.7 and $0.55 \mathrm{~dB}$ at $60{ }^{\circ} \mathrm{C}$ and $-10{ }^{\circ} \mathrm{C}$, respectively, because the micromirror will cause additional insertion loss if it is not at the center of the flap. On the other hand, the TDL is minimized if the micromirror is lined up at the center of the actuation flap. Fig. 11(c) shows the measurement of ten temperature cycles, and the TDL can be as large as $0.3 \mathrm{~dB}$ when the micromirror is not at the center of the flap. The mirror position is mainly determined by the double-sided alignment of the aligner, and the accuracy can be controlled within $\pm 5 \mu \mathrm{m}$, which is less than the thickness of a micromirror of $20 \mu \mathrm{m}$. The measured TDL is less than $0.05 \mathrm{~dB}$ for a well-aligned micromirror.

Because the reflectivity of the micromirror is almost independent of the light wavelength, we expect that the wavelength dependence loss (WDL) is low. The WDL is measured by a broadband light source and optical spectrum analyzer. The WDL of a micromirror is $0.05 \mathrm{~dB}$ with a wavelength span of $100 \mathrm{~nm}$ (from 1510 to $1610 \mathrm{~nm}$ ), which covers both the $C$-band and $L$-band, as shown in Fig. 12. We believe that this low WDL is partially attributed to the noncurved SCS mirror surface.

The polarization dependence loss (PDL) is measured by an Agilent 8509B with Jones matrix calculation, and the laser source is from an Agilent 81642A tunable laser, which covers the wavelength from 1510 to $1600 \mathrm{~nm}$. The PDL of a micromirror is less than $0.1 \mathrm{~dB}$, including the effect of collimators, as plotted in Fig. 13. 


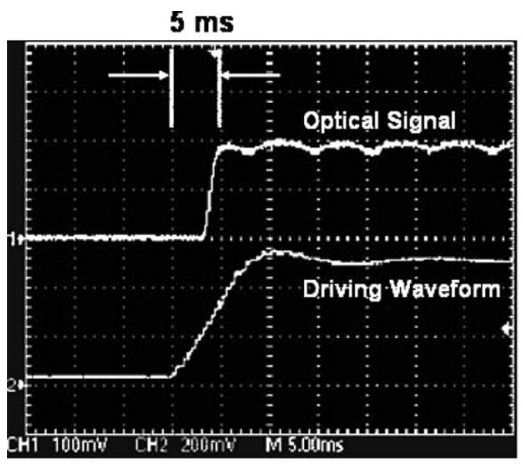

Switching On

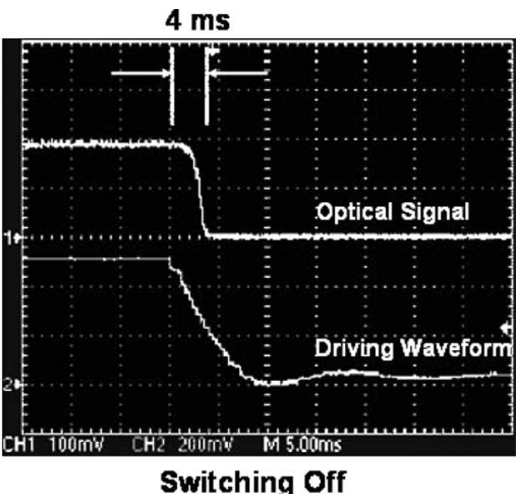

Switching Off

Fig. 14. Switching time of a 2-D MEMS switch.

Regarding the vibration, specially designed serpentine springs are applied to the optical switch, and the experimental results show that the device could pass the severe Telcordia GR-1221 requirement during the transportation, which requires a $20-\mathrm{G}$ acceleration with a sweeping frequency in the range of $10-2000 \mathrm{~Hz}$ [13]. During operation, we vibrated the device at $1-\mathrm{G}$ acceleration with the frequency ranging from 0.1 to $100 \mathrm{~Hz}$, and we observed that the optical signal ripples were less than $0.1 \mathrm{~dB}$. However, the ripples increased to more than $1 \mathrm{~dB}$ when the vibration frequency was close to its resonant frequency, which is around $125 \mathrm{~Hz}$.

The flap is moving in the air and is not in contact with any mechanical parts. This design could eliminate possible stiction issues, but this makes fast switching a challenge. The switch is driven by a preshaping waveform stored in the control circuit board, and the switching time is less than $5 \mathrm{~ms}$, as shown in Fig. 14. To achieve short switching time, we need to take care of the resonant frequency of the actuation flap. The special waveform is edited based on the frequency response of the actuation flap. The special waveform is started with a basic step function where the resonant-frequency components of the actuation flap are reduced to eliminate the overshooting of vertical micromirrors. The speed of switching on and off is 5 and $4 \mathrm{~ms}$, respectively.

\section{CONCLUSION}

A novel MEMS fabrication process was developed to integrate vertical stress-free micromirrors and electromagnetic actuators without an extra assembly process. The size of the TMAH etched micromirror is $20 \mu \mathrm{m} \times 500 \mu \mathrm{m} \times 1200 \mu \mathrm{m}$, which is large enough to ensure low insertion loss for 2-D optical-switching applications. The surface roughness of the micromirror is measured to be as low as $20 \mathrm{~nm}$ by the white light interferometer, which typically results in $0.2-\mathrm{dB}$ optical loss. The center-aligned vertical mirror showed an excellent TDL of $0.05 \mathrm{~dB}$. An appropriate magnetic circuit design reduces the actuation voltage to $0.5 \mathrm{~V}$ with a power consumption of about $3.5 \mathrm{~mW}$. A reasonable fast-switching time of $5 \mathrm{~ms}$ can be achieved by eliminating the resonant-frequency components of the MEMS switch through edited waveforms. Long-term cycling and mechanical reliability tests proved that the proposed MEMS optical switch can pass the rigorous requirement of optical communications. The PDL and WDL are both less than $0.1 \mathrm{~dB}$. The design, fabrication, and tests of low-actuationvoltage MEMS switches have been presented in this paper.

\section{REFERENCES}

[1] L. Lin and E. Goldstein, "Opportunities and challenges for MEMS in lightwave communications," IEEE J. Sel. Topics Quantum Electron., vol. 8, no. 1, pp. 163-172, Jan./Feb. 2002.

[2] X. Ma and G. Kuo, "Optical switching technology comparison: Optical MEMS versus other technologies," IEEE Commun. Mag., vol. 41, no. 11, pp. S16-S23, Nov. 2003.

[3] D. Bishop, C. Giles, S. Das et al., "The rise of optical switching," Sci. Amer., vol. 284, no. 1, pp. 88-94, Jan. 2001.

[4] A. Neukermans and R. Ramaswami, "MEMS technology for optical networking applications," IEEE Commun. Mag., vol. 39, no. 1, pp. 62-69, Jan. 2001

[5] P. Dobbelaere, K. Falta, L. Fan, S. Gloeckner, and S. Patra, "Digital MEMS for optical switching," IEEE Commun. Mag., vol. 40, no. 3, pp. 88-95, Mar. 2002.

[6] L. Lin, E. Goldstein, and R. Tkach, "On the expandability of the freespace micromachined optical cross connects," J. Lightw. Technol., vol. 18, no. 4, pp. 482-489, Apr. 2000

[7] C. Marxer, C. Thio, M. Gretillat, N. F. de Rooji, R. Battig, R. Anthamatten, B. Valk, and P. Vogel, "Vertical mirrors fabricated by deep reactive ion etching for fiber-optic switching applications," J. Microelectromech. Syst., vol. 6, no. 7, pp. 277-285, Jul. 1997.

[8] R. T. Chen, H. Nguyen, and M. C. Wu, "A high-speed low-voltage stressinduced micromachined $2 \times 2$ optical switch," IEEE Photon. Technol. Lett., vol. 11, no. 11, pp. 1396-1398, Nov. 1999.

[9] G. Su, H. Toshiyoshi, and M. C. Wu, "Surface-micromachined 2-D optical scanners with high-performance single-crystalline silicon micromirrors," Photon. Technol. Lett., vol. 13, no. 6, pp. 606-608, Jun. 2001.

[10] J. W. Judy, "Microelectromechanical Systems (MEMS) - Their design, fabrication, and broad range of application," J. Smart Mater, vol. 10, no. 6 , pp. 1115-1134, Dec. 2001.

[11] L. Houlet, P. Helin, T. Bourouina, G. Reyne, E. Gergam, and H. Fujita, "Movable vertical mirror arrays for optical microswitch matrixes and their electromagnetic actuation," IEEE J. Sel. Topics Quantum Electron., vol. 8, no. 1, pp. 58-63, Jan./Feb. 2002.

[12] Telcordia GR-1073-CORE, pp. 4-11, Jan. 2001. Issue 1.

[13] G. D. J. Su, S. H. Hung, D. Jia, and F. Jiang, "Serpentine spring corner design for MEMS optical switches with large mirror mass," Opt. Rev., vol. 12, no. 4, pp. 339-344, Jul./Aug. 2005.

[14] E. M. Conway and V. J. Cunnane, "Electrochemical characterization of $\mathrm{Si}$ in tetra-methyl ammonium hydroxide (TMAH) and TMAH: TritonX-100 solutions under white light effects," J. Micromech. Microeng., vol. 12, no. 2, pp. 136-148, Mar. 2002.

Hsin-Ta Hsieh received the B.S. degree in physics from the National ChengKung University, Tainan, Taiwan, R.O.C., and the M.S. degree from the Graduate Institute of Electro-Optical Engineering, National Taiwan University, Taipei, Taiwan. He is currently working toward the Ph.D. degree at the Graduate Institute of Electro-Optical Engineering, National Taiwan University.

His research interests include microelectromechanical-systems-based optical components, especially optical switches for fiber communication. 
Chen-Wei Chiu received the B.S. degree in mechanical engineering from National Taiwan University, Taipei, Taiwan, R.O.C., in 1994 and the M.S. degree from the University of California, Los Angeles, in 1998.

$\mathrm{He}$ is a Cofounder of Umachines, Inc., Altadena, CA, and still contributes his expertise in the optical microelectromechanical systems field through a collaboration with National Taiwan University.

Tom Tsao received the Ph.D. degree in electrical engineering from California Institute of Technology, Pasadena, in 1998.

He worked on the actuator, for use in control and on integrating the shear stress sensor, actuator, and electronics all on one substrate. He is a Cofounder and the President of Umachines, Inc., Altadena, CA.
Fukang Jiang received the Ph.D. degree in electrical engineering from California Institute of Technology, Pasadena, in 1998.

$\mathrm{He}$ is a Cofounder and the Vice President of Engineering of Umachines, Ltd., Altadena, CA. His research interests include the development of microelectromechanical-systems flow sensors and flexible skins with integrated sensors and electronics.

Guo-Dung John Su (M'02) received the B.S. degree from National Taiwan University, Taipei, Taiwan, R.O.C., in 1994, and the M.S. and Ph.D. degrees in electrical engineering from the University of California, Los Angeles, in 1998 and 2001, respectively. His doctoral research interest was related to microelectromechanical systems (MEMS) scanners with flat mirror surface for active optical alignment and micromirror arrays for adaptive-optics applications.

Since 2004, he has been an Assistant Professor with the Graduate Institute of Electro-Optical Engineering and the Department of Electrical Engineering, National Taiwan University. His current research interests include MEMS devices for optical communications, compact optical imaging systems, and surface plasmon phenomenon on nanoparticles. 